\title{
Intrauterine Corticosteroids for Lung Maturation: Observations of HPA-Axis Function and Cardiac Autonomic Balance in the Human Neonate
}

\author{
Leonhard Schäffer, Tilo Burkhardt, Ernst Beinder
}

Department of Obstetrics and Gynecology, University Hospital of Zurich, Switzerland

The putative long term effects of antenatal glucocorticoid treatment for accelerating lung maturation in pregnancies at risk for preterm birth is still a matter of debate. Different animal models have shown that antenatal glucocorticoid exposure has a persistent impact on the hypothalamus-pituitary-adrenal (HPA) - axis regulation and that the developing cardiac autonomic system is sensitive towards antenatal glucocorticoids. In the human, the situation is less clear. Long term observations in adolescents and young adults have rather focused on classic signs of disease manifestation such as blood pressure. Risk factors for cardiovascular and metabolic disease, however, may transform into apparent clinical manifestation at a more advanced age when stability of these systems can be less compensated by the organism. We analysed resting- and stress-induced salivary levels for cortisol, cortisone and $\alpha$-amylase as well as heart rate variability parameters as early signs of system alteration to find out whether a single course of antenatal betamethasone treatment has a direct and continuous effect on the developing HPA axis and the cardiac autonomic system. We conducted our study in the neonatal period in healthy newborns born late preterm or at term to find out whether putative intrauterine steroid effects ultimately transform into permanent postnatal alterations and to exclude postnatal induced influencing factors of system adaptation. Our results show that a single course of antenatal betamethasone treatment induces a significant suppression of HPA-axis reactivity in healthy infants that is present several weeks later in early postnatal life. By contrast, the cardiac autonomic balance is preserved. Antenatal betamethasone treatment may therefore alter the HPA axis regulation in a manner that predisposes these individuals towards the development of diseases in later life.

\footnotetext{
* Schäffer L, Burkhardt T, Tomaske M, Rauh M, Beinder E, Intrauterine corticosteroids for lung maturation. Observations of HPA axis function and cardiac autonomic balance in the neonate. In: Plagemann A, editor. Perinatal programming - The State of the Art. Berlin: Walter de Gruyter, 2011.
} 